\title{
Amaurosis fugax: some aspects of management
}

\author{
PJ PARKIN, BE KENDALL, J MARSHALL, WI MCDONALD
}

From the National Hospital for Nervous Diseases, Queen Square and Maida Vale, and the Moorfields Eye Hospital, London

SUMMARY Fifty-one patients with amaurosis fugax were followed for a mean of 4.9 years from their first attack. Females predominated under the age of 50 years in contrast to men who presented in the older age group. Minor permanent visual sequelae occurred in only three patients, but cerebral vascular symptoms were present at some time in just under half the group. A range of abnormalities was identified on cerebral angiography and some of these correlated with certain clinical findings. Twenty patients were treated by surgery and permanent relief or significant improvement in symptoms occurred in 14. A group of patients who were at greater risk of cerebral vascular complications following angiography and surgery was identified. It is concluded that careful patient selection is necessary before surgery is recommended but that in a significant number of cases with relatively focal atheroma confined to one proximal internal carotid artery an excellent long term result following surgery may be expected.

Descriptions of amaurosis fugax are on record since the early 1800s but it was not until as recently as nearly 30 years ago that Fisher drew attention to the association of transient attacks of monocular blindness with contralateral hemiplegia. ${ }^{1}$ Since then, radiological and pathological experience has convincingly confirmed the relationship of amaurosis fugax to atheromatous disease of the carotid artery and to the risk of stroke. ${ }^{2}$ The natural history of amaurosis fugax has been most clearly defined by Marshall and Meadows in 1968, when they followed 80 patients up to four years after the onset of symptoms. Permanent monocular blindness, complete hemisphere stroke or both followed an attack of amaurosis fugax in $16 \% \%^{3}$ A surgical approach to therapy was therefore advocated by Ehrenfeld and others when they described the outcome of 44 patients with amaurosis fugax. Of 12 unoperated patients reviewed retrospectively, 10 had developed stroke or permanent blindness. Nearly all the cases treated by carotid endarterectomy were relieved of symptoms. ${ }^{4}$ More recently, Mungas and Baker treated 80 patients by carotid endarterectomy.

Address for reprint requests: Professor WI McDonald, The National Hospital, Queen Square, London WCIN 3BG, UK.

Received 5 October 1981

Accepted 8 October 1981
Relief of symptoms occurred in $90 \%$ but the mean length of follow-up was little over one year. ${ }^{5}$

We have recently reviewed our own experience of managing patients with amaurosis fugax over a seven year period. Although we have reviewed all patients presenting in that time we have paid particular attention to establishing the outcome of those treated by surgery.

\section{Clinical material}

Fifty-six patients were seen over a seven year period from 1973. They were referred from the clinics of an ophthalmological hospital and a general hospital, from physicians in other centres and by direct referral from family practitioners. Patients whose visual symptoms may have been attributable to primary ophthalmologic disease or to intracerebral disease have not been considered and patients with pre-existing monocular visual impairment have also been excluded. All patients had experienced at least one attack of transient monocular blindness. For the purposes of this study amaurosis fugax was defined as partial or complete transient monocular blindness with total recovery within 24 hours. In fact, the duration of visual loss was rarely longer than a few minutes in the great majority. In all patients the frequency of attacks over the period of follow-up was established and particular attention was paid to the occurrence of symptoms suggesting transient cerebral ischaemic attacks (TIA), peripheral vascular disease and coronary heart disease. All patients underwent clinical re-examination. Cerebral angiograms performed during the original admission were reviewed. 


\section{Results}

One patient had died of carcinoma of the pancreas. Four others could not be traced and their fate is unknown. Fifty-one patients were reviewed, a mean 4.9 years from the onset of their first attack and a mean of four years from the time of presentation. Seven of the patients had a history of either TIA or completed stroke prior to developing amaurosis fugax and they have been followed for a mean of $5 \cdot 1$ years from the first cerebral vascular symptom.

\section{SEX INCIDENCE}

The sex incidence in the whole group showed a male predominance of $2 \cdot 6: 1$. Females, however, more commonly presented under the age of 50 years in contrast to males who showed a striking predominance over that age (table 1).

Table 1 Distribution of patients according to age and sex

\begin{tabular}{lccc}
\hline Age & Males & Females & Total \\
\hline $30-39$ & 2 & 1 & 3 \\
$40-49$ & 4 & 9 & 13 \\
$50-59$ & 16 & 1 & 17 \\
$60-69$ & 12 & 3 & 15 \\
70.79 & 3 & 0 & 3 \\
Total & 37 & 14 & 51 \\
\hline
\end{tabular}

PERMANENT VISUAL SEQUELAE

Persisting visual sequelae occurred in three patients. A sector field defect was present in all and in two of them an impacted retinal embolus was visible. In no patient was the peripheral field defect a major visual handicap. The attacks of amaurosis fugax had lasted a few minutes in one patient, 30-60 minutes in another and in the third patient some attacks had lasted for up to 12 hours.

\section{CEREBRAL VASCULAR SYMPTOMS}

Cerebral vascular symptoms (TIA or stroke) occurred in 22 patients $(43 \%)$, either preceding or following amaurosis fugax (table 2). Of 44 patients in the series who were without cerebral ischaemic symptoms prior to the onset of amaurosis fugax, $15(34 \%)$ subsequently developed TIA or stroke.

Table 2 Associated cerebral vascular symptoms in 22 patients with amaurosis fugax

\begin{tabular}{lr}
\hline Cerebral vascular symptoms- & 7 \\
Before amaurosis & 11 \\
Following amaurosis & 1 \\
Simultaneous TIA and amaurosis & 1 \\
Simultaneous stroke and amaurosis & 2 \\
Asymptomatic focal signs & Total \\
& 22 \\
\hline
\end{tabular}

RETINAL EMBOLI

Retinal emboli were present at some time in eight patients (seven at the time of presentation; one at follow-up). In six, the emboli were refractile yellow crystals and in two the nature is uncertain. Two were associated with retinal arteriolar branch occlusion. All but one patient had an ipsilateral carotid bruit. Coronary heart disease or peripheral vascular disease was present in five. All four patients undergoing cerebral angiography had a localised atheromatous lesion suitable for carotid endarterectomy. One patient in this group, who received no therapy, developed stroke and one patient developed TIA responding to antiplatelet therapy.

\section{MULTIPLE ATTACKS}

Multiple attacks, defined as greater than 20 prior to presentation, had occurred in 13 patients. Although they received a variety of treatments, none had developed permanent visual sequelae by the time of follow-up. Carotid territory TIAs had occurred before visual symptoms in two patients and a further three developed TIAs later.

BILATERAL ATTACKS

In five patients attacks of amaurosis fugax were independently bilateral. Bilateral angiography was performed in four of them and a localised unilateral proximal internal carotid lesion was present in three. All four angiograms, however, showed atheromatous irregularity of variable degree on both sides. One patient later developed stroke; one developed TIAs that persisted despite unilateral carotid endarterectomy and later treatment with anticoagulants; and in two patients the attacks ceased spontaneously and the patients remain well.

\section{ASSOCIATED DISORDERS}

An elevation in the fasting level of serum cholesterol or triglycerides above $6.5 \mathrm{mmol} / \mathrm{l}$ or $1.7 \mathrm{mmol} / \mathrm{l}$ respectively was present in 31 patients $(61 \%)$. The diastolic blood pressure exceeded $100 \mathrm{~mm} \mathrm{Hg}$ in nine patients $(18 \%)$. A family history of ischaemic heart disease, peripheral vascular disease or stroke under the age of 60 years was present in 19 patients $(37 \%)$. An abnormality of the electrocardiogram was present in six; five showed ischaemic or hypertensive changes and one showed atrial fibrillation. Clinical evidence of ischaemic heart disease or peripheral vascular disease was noted at presentation in 13 patients $(25 \%)$ and at follow-up in an additional nine patients $(18 \%)$.

CEREBRAL ANGIOGRAPHY

Thirty-eight patients underwent cerebral angiography, a greater proportion of patients under the age of 50 
years being investigated in this way (14 of 16 patients). Carotid angiography was performed initially on the symptomatic side and the contralateral carotid examined only if a potentially surgically treatable lesion had been shown. All studies were performed under general anaesthesia with controlled ventilation. Catheterisation via a transfemoral approach was the procedure most commonly used. Direct common carotid puncture alone was the method employed in some cases, but was also used to supplement the former examination if difficulty with selective catheterisation was encountered. Thus, half the patients studied by angiography were subjected to carotid puncture.

In the 24 patients over the age of $50 \mathrm{yr}$, angiography demonstrated irregularity or stenosis of the proximal internal carotid artery suitable for endarterectomy in $16(67 \%)$. By contrast this abnormality was present in only five of 14 angiograms $(36 \%)$ performed in the younger age group. Vessel wall irregularity, however, was generally more widespread than this and all but four patients showed additional disease of variable severity in the distal carotid circulation on the same side and all but two showed disease at some point in the contralateral carotid circulation. The presence of certain clinical features in the patients showing a carotid lesion suitable for surgery are listed in table 3 . Of the 21 cases with an operable carotid lesion, only three did not have an ipsilateral carotid bruit or hypertension or both, and in the under 50 year age group no patient was without one of these signs.

Table 3 Clinical signs and the presence of a promixal internal carotid lesion suitable for surgery. From 38 patients studied by angiography

\begin{tabular}{lll}
\hline & Number of patients & $\begin{array}{l}\text { Number with a "surgical" } \\
\text { lesion }\end{array}$ \\
\hline Bruit & 14 & 12 \\
TIA or stroke & 16 & 10 \\
Hypertension & 7 & 5 \\
Age under 50 & 14 & 5 \\
Age over 50 & 24 & 15 \\
\hline
\end{tabular}

In addition to the 21 angiograms described above, angiography demonstrated complete carotid occlusion in four patients (one of whom also had a surgically treatable lesion on the other side); severe focal irregularity of the carotid siphon (one patient); mild proximal internal carotid irregularity (five patients); minimal carotid irregularity (seven patients); and no abnormality (one patient). The ophthalmic artery failed to fill in two patients. In the angiograms of three patients, retrograde filling of the ophthalmic artery by collateral vessels from the external carotid artery was present and this was associated with high grade stenosis of the proximal internal carotid artery in two and with complete carotid occlusion in one.

Angiography was associated with complications in three patients. One of them, aged 50 years, had already experienced a minor stroke before presentation and this was made worse following angiography and a moderate disability persists. Two others, aged 45 and 61 years, sustained minor strokes within 24 hours of angiography; one recovered completely and the other was left with a mild residual disability. Complete internal carotid artery occlusion was present in two of these patients and a severe stenosis of the carotid siphon was present in the third. In all three cases angiography had been performed by direct common carotid puncture.

\section{PATIENTS TREATED BY SURGERY}

Twenty patients underwent surgery. Carotid endartercetomy was performed on 19 and one patient was treated by extracranial-intracranial anastomosis as the primary procedure. A number of the patients continued to receive prophylactic antiplatelet aggregation therapy after operation.

Four patients underwent more than one operation. One experienced a recurrence of amaurosis fugax and TIAs 10 months after endarterectomy. Angiography showed a re-stenosis of the internal carotid artery at its origin and a second endarterectomy was followed by abolition of symptoms for $2 \frac{1}{2}$ years before they returned. A second patient had a critical proximal internal carotid stenosis demonstrated by angiography but had developed carotid occlusion by the time of operation and because of this extracranialintracranial anastomosis was performed one week later. A third patient required external carotid endarterectomy following endarterectomy of the internal carotid because of continuing symptoms. The fourth patient experienced no improvement in symptoms following internal carotid endarterectomy and underwent the same procedure on the contralateral side 4 months later.

The average age of the surgically treated patients was 56.5 years and the average duration of follow-up from the time of surgery is $\mathbf{4 . 0}$ years. Eleven patients were relieved of all symptoms, including two with TIA and three with multiple attacks of amaurosis fugax. Three further patients had a significant reduction in the frequency of attacks following surgery. One of these had a single recurrence of amaurosis 4 months after surgery but remains well 7 years later; one was relieved of symptoms for 3 years before amaurosis fugax recurred; in one patient the attacks were abolished for 10 months before they recurred and re-endarterectomy again abolished symptoms for $2 \frac{1}{2}$ years. 
In two patients the frequency of attacks was unchanged by surgery, each patient having experienced both multiple attacks of amaurosis fugax and TIAs. They were also unresponsive to anticoagulant and antiplatelet therapy. One patient was worse as result of surgery, having developed carotid thrombosis by the time of operation and this resulted in a mild persisting hemiparesis. In three patients the frequency of attacks was reduced for a period following surgery but they developed stroke 8-28 months later in the same carotid territory. A history of TIA had been present in two of them prior to operation. In one, the internal carotid artery on the symptomatic side was completely occluded and endarterectomy was performed for a stenosis on the other side. The second patient had, in addition to a proximal internal carotid stenosis, severe disease in the ipsilateral carotid siphon. In the third patient, angiography had been interpreted as showing complete occlusion of the internal carotid with retrograde distal filling from the ophthalmic artery, and she underwent extracranial-intracranial anastomosis as the primary procedure. Our review of the serial subtraction films from this angiogram revealed that progressive retrograde filling of the distal internal carotid occurred until it encountered a profoundly slowed anterograde flow from the proximal internal carotid artery. Thus the carotid system was in fact patent although the profound slowing in blood flow resulting from a critical proximal stenosis had simulated internal carotid occlusion (fig).

Four other patients experienced minor sequelae following surgery. Two had minor completed strokes which recovered within 1-2 weeks; one complained of persistent pain about the operation site; one developed a transient hypoglossal palsy.

\section{Discussion}

The importance of amaurosis fugax as a marker of carotid arterial disease is emphasised by the experi-

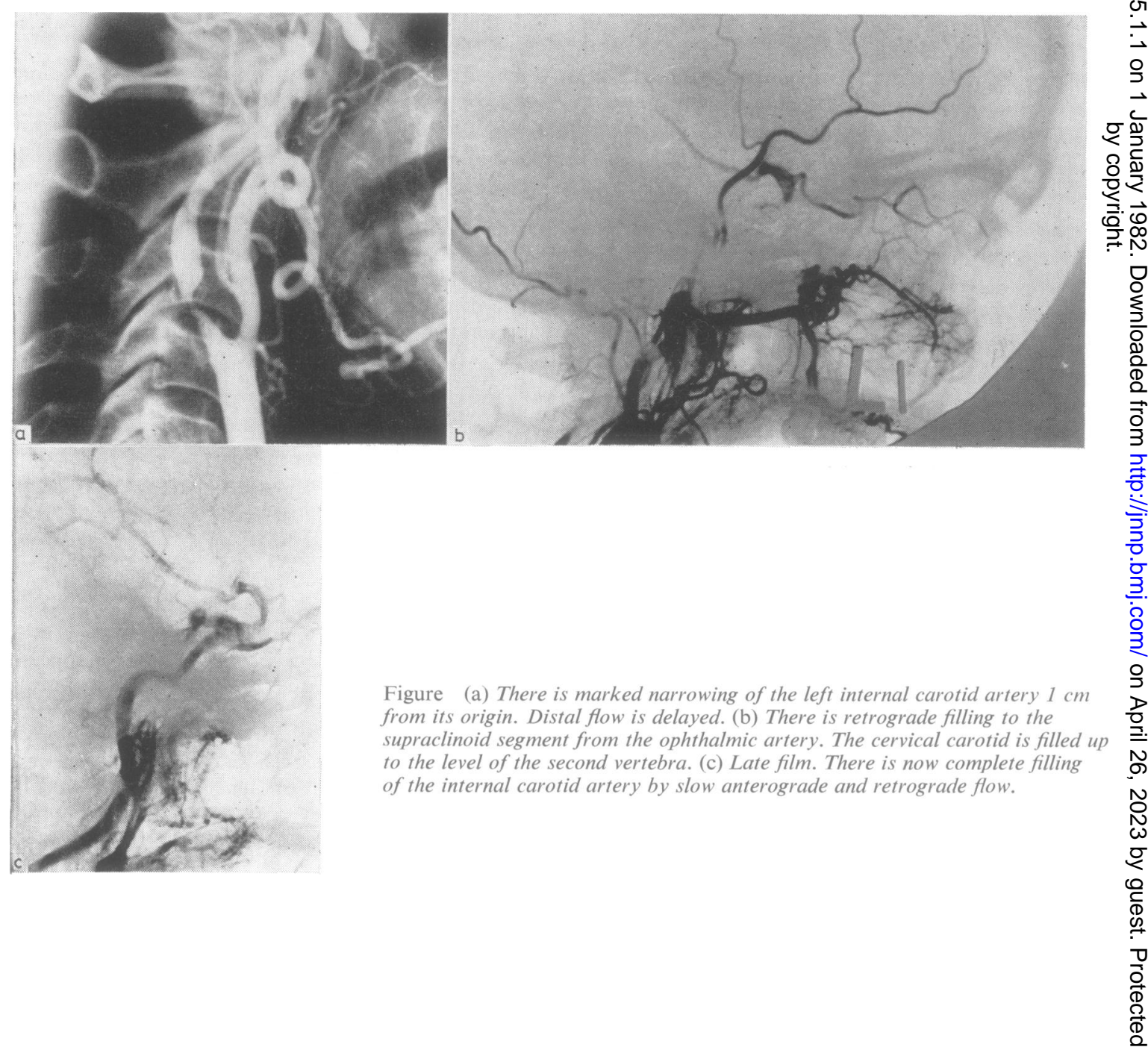


ence of our study. When considered without regard to therapy, just under half the patients in our series had developed TIA or stroke within the period of follow-up. Amaurosis fugax may also be a marker of more generalised disease as evidenced by the fact that just under half had developed peripheral vascular disease or ischaemic heart disease by the time of follow-up.

The neurological prognosis in the individual who presents with isolated amaurosis fugax is difficult to predict on clinical grounds. Neither the frequency nor the duration of the attacks seemed to bear any direct relationship to the risk of developing cerebral vascular complications and this was also the experience of Marshall and Meadows. ${ }^{3}$ Permanent visual sequelae occurred in only three patients in our series and in two of them the duration of individual attacks of amaurosis fugax was longer than the few minutes that characterised attacks in the majority of patients. However, in the small number in whom the attacks did last for a longer period the risk of cerebral complications was no greater than in the group as a whole, and in patients experiencing multiple attacks no increase in risk was evident. Indeed, the prognosis in individual patients was quite variable. Even in those experiencing attacks which were multiple, of prolonged duration, present for a considerable period, or were bilateral, spontaneous remission of symptoms without treatment sometimes occurred.

In the group of patients under the age of 50 years, females predominated. Although it is possible that aetiological factors other than atheromatous vascular disease might account for symptoms in a proportion, over one third experienced TIA and somewhat under half had either proximal carotid atheroma sufficient to justify endarterectomy, or complete carotid occlusion. In women, amaurotic attacks might arise through migraine or hormonal drug ingestion but only one of 10 women was taking an hormonal contraceptive and in no patient was migraine considered a likely explanation.

The high incidence of carotid arterial disease in patients with amaurosis fugax was confirmed by angiography. Only $21 \%$ of patients undergoing angiography showed no abnormality or only minimal vessel wall irregularity. The remainder all showed atheromatous irregularity, stenosis or complete vessel occlusion at some part of the carotid circulation and the range of angiographic abnormalities was similar to that found by others. ${ }^{6-8}$ The likelihood of a lesion in the region of the proximal internal carotid artery was increased if the patient displayed a localised carotid bruit, had an associated history of TIA or stroke, was hypertensive or was in the older age group and this experience is similar to that reported by Wilson and Ross Russell.?
Emboli arising from proximal internal carotid lesions may cause symptoms by entering the ophthalmic or cerebral circulations and this is the rationale behind treatment by carotid endarterectomy. In the patients who had complete occlusion of the internal carotid artery symptoms may have been produced by different mechanisms. Emboli may arise from the stump of the internal carotid artery to enter the external carotid artery and travel to the eye or brain by collateral vessels. ${ }^{9}{ }^{10}$ Patients who, in addition to internal carotid occlusion, have disease in the external carotid may develop symptoms when emboli arise from the external carotid artery itself. ${ }^{11}$ Retrograde flow through the ophthalmic artery may result in ophthalmic "steal" when this vessel provides an anastomotic channel by which the external carotid circulation is able to supply the distal internal carotid artery to bypass an occlusion. In the two patients in whom the ophthalmic artery failed to fill on angiography, symptoms may have arisen as a result of diminished flow through the vessel or by embolisation from an ophthalmic arterial stenotic lesion. ${ }^{12}$

Because of the clinical and radiological differences between patients treated medically and those treated surgically no attempt was made in this study to compare the outcome of patients in different treatment groups. In the patients treated by surgery an excellent result, with relief of all symptoms, was achieved in just over half ( 11 of 20 patients) and improvement in symptoms occurred in a further three patients. In just under one third of the surgical group (six patients) the long term results were less satisfactory, although only one patient was permanently worse as a direct consequence of surgery. Two of these patients experienced no change in symptoms and three patients developed a stroke at some later date. The three patients who developed late stroke were poor risk cases. In one of them, endarterectomy was undertaken on the side contralateral to a carotid occlusion. In another, severe additional disease in the carotid siphon was not able to be treated surgically and may have been responsible for later stroke. The third patient had been treated by extracranial-intracranial anastomosis for what had been thought to be an internal carotid occlusion. Review of the angiogram, however, indicated that a proximal internal carotid stenosis, although critical, was not in fact complete and carotid endarterectomy was probably the primary procedure of choice. This case does emphasise the importance of detailed examination of serial films of the entire angiographic series in patients with apparently complete internal carotid occlusion.

The one patient who was rendered worse immediately following surgery had a high grade 
stenosis of the proximal internal carotid and lesions such as this carry a risk of complete thrombosis by the time surgery is performed. In the two patients who sustained minor cerebral vascular complications following surgery the recovery was complete within two weeks.

The fact that three of the 20 patients who underwent surgery experienced a late stroke might cast doubt on the efficacy of endarterectomy as a prophylactic measure. However, as already mentioned, these three patients had extensive disease. This experience emphasises the importance of careful patient selection before surgery is recommended. Patients who have a critical stenosis of the proximal internal carotid, contralateral carotid occlusion, disease of the distal carotid circulation, or who have atheromatous disease in both carotid circulations probably carry a much greater surgical risk. It is in these cases in particular that increased risks must be carefully weighed against the risks inherent in the disease itself. However, in patients with relatively focal atheromatous disease confined to one proximal internal carotid, our experience suggests that a significant proportion will have their symptoms abolished at an acceptably low risk.

We are grateful to the physicians and surgeons who referred the patients to us, and to Professor G Taylor and to $\mathbf{M r} \mathbf{J}$ Lumley who were responsible for the surgery.

\section{References}

${ }^{1}$ Fisher CM. Transient monocular blindness associated with hemiplegia. Arch Ophthalmol 1952;47:167-203.

${ }^{2}$ Gunning AJ, Pickering GW, Robb-Smith AHT, Ross Russell R. Mural thrombosis of the internal carotid artery and subsequent embolism. $Q J$ Med 1964;33: 155-95.

${ }^{3}$ Marshall J, Meadows S. The natural history of amaurosis fugax. Brain 1968;91:419-53.

${ }^{4}$ Ehrenfeld WK, Hoyt WF, Wylie EJ. Embolization and transient blindness from carotid atheroma. Arch Surg 1966;93:787-94.

${ }^{5}$ Mungas JE, Baker WH. Amaurosis fugax. Stroke 1977; 8:232-5.

${ }^{6}$ Harrison MJ, Marshall J. Indications for angiography and surgery in carotid artery disease. $\mathrm{Br}$ Med $\mathrm{J}$ 1975; 1 :616-8.

'Wilson LA, Ross Russell RW. Amaurosis fugax and carotid artery disease: indications for angiography. Br Med J 1977;2:435-7.

${ }^{8}$ Eisenberg RL, Main RL. Clinical and arteriographic comparison of amaurosis fugax with hemispheric transient ischaemic attacks. Stroke 1978;9:254-5.

${ }^{9}$ Barnett HJM, Peerless SJ, Kaufmann JCE. "Stump" of the internal carotid artery-a source for further cerebral embolic ischaemia. Stroke 1978;9:448-56.

${ }^{10}$ Countee RW, Vijayanathan T. Intracranial embolisation via external carotid artery: report of a case with angiographic documentation. Stroke 1980;11:465-8.

${ }^{11}$ Burnbaum MD, Selhorst JB, Harbison JW, Brush JJ. Amaurosis fugax from disease of the external carotid artery. Arch Neurol 1977;34:532-5.

${ }^{12}$ Weinberger J, Bender AN, Yang WC. Amaurosis fugax associated with ophthalmic artery stenosis: clinical simulation of carotid artery disease. Stroke $1980 ; 11: 290-3$. 